\title{
Cerenkov Luminescence Imaging of Medical Isotopes
}

\author{
Alessandro Ruggiero*1, Jason P. Holland*2, Jason S. Lewis ${ }^{2,3}$, and Jan Grimm ${ }^{1,3}$ \\ ${ }^{I}$ Nuclear Medicine Service, Department of Radiology, Memorial Sloan-Kettering Cancer Center, New York, New York; \\ ${ }^{2}$ Radiochemistry Service, Department of Radiology, Memorial Sloan-Kettering Cancer Center, New York, New York; \\ and ${ }^{3}$ Molecular Pharmacology and Chemistry Program, Memorial Sloan-Kettering Cancer Center, New York, New York
}

The development of novel multimodality imaging agents and techniques represents the current frontier of research in the field of medical imaging science. However, the combination of nuclear tomography with optical techniques has yet to be established. Here, we report the use of the inherent optical emissions from the decay of radiopharmaceuticals for Cerenkov luminescence imaging (CLI) of tumors in vivo and correlate the results with those obtained from concordant immuno-PET studies. Methods: In vitro phantom studies were used to validate the visible light emission observed from a range of radionuclides including the positron emitters ${ }^{18} \mathrm{~F},{ }^{64} \mathrm{Cu},{ }^{89} \mathrm{Zr}$, and ${ }^{124} \mathrm{I} ; \beta$-emitter ${ }^{131} \mathrm{I}$; and $\alpha$-particle emitter ${ }^{225} \mathrm{Ac}$ for potential use in CLI. The novel radiolabeled monoclonal antibody ${ }^{89} \mathrm{Zr}$-desferrioxamine $\mathrm{B}$ [DFO]-J591 for immuno-PET of prostate-specific membrane antigen (PSMA) expression was used to coregister and correlate the CLI signal observed with the immuno-PET images and biodistribution studies. Results: Phantom studies confirmed that Cerenkov radiation can be observed from a range of positron-, $\beta-$, and $\alpha$-emitting radionuclides using standard optical imaging devices. The change in light emission intensity versus time was concordant with radionuclide decay and was also found to correlate linearly with both the activity concentration and the measured PET signal (percentage injected dose per gram). In vivo studies conducted in male severe combined immune deficient mice bearing PSMA-positive, subcutaneous LNCaP tumors demonstrated that tumor-specific uptake of ${ }^{89} \mathrm{Zr}$-DFO-J591 could be visualized by both immuno-PET and CLI. Optical and immuno-PET signal intensities were found to increase over time from 24 to $96 \mathrm{~h}$, and biodistribution studies were found to correlate well with both imaging modalities. Conclusion: These studies represent the first, to our knowledge, quantitative assessment of $\mathrm{CLI}$ for measuring radiotracer uptake in vivo. Many radionuclides common to both nuclear tomographic imaging and radiotherapy have the potential to be used in CLI. The value of CLI lies in its ability to image radionuclides that do not emit either positrons or $\gamma$-rays and are, thus, unsuitable for use with current nuclear imaging modalities. Optical imaging of Cerenkov radiation emission shows excellent promise as a potential new imaging modality for the rapid, high-throughput screening of radiopharmaceuticals.

Received Feb. 19, 2010; revision accepted Mar. 19, 2010. For correspondence or reprints contact: Jan Grimm, Molecular Pharmacology and Chemistry Program, Memorial Sloan-Kettering Cancer Center, 1275 York Ave., New York, NY 10065.

E-mail: grimmj@mskcc.org

${ }^{*}$ Contributed equally to this work.

COPYRIGHT () 2010 by the Society of Nuclear Medicine, Inc.
Key Words: imaging technology; Cerenkov; PET; optical imaging; ${ }^{89} \mathrm{Zr}$; ${ }^{124} \mathrm{I}$; ${ }^{131} \mathrm{I} ;{ }^{64} \mathrm{Cu}$; ${ }^{225} \mathrm{Ac}$; ${ }^{18} \mathrm{~F}$; radioimmunoconjugate; prostate-specific membrane antigen (PSMA); J591; monoclonal antibodies

J Nucl Med 2010; 51:1123-1130

DOI: 10.2967/jnumed.110.076521

$n$ the field of medical imaging science, the concept of multimodality is providing the driving force for the development of the next generation of imaging techniques. The latest hybrid systems such as PET/CT and PET/MRI are transforming the clinical management of cancer patients by consolidating the noninvasive localization and temporal quantification of changes in tissue function and physiology available from PET, with the high-resolution anatomic maps provided by CT or MRI $(1,2)$.

In contrast to the immediate clinical impact of nuclear tomographic imaging, optical methods such fluorescencemediated tomography and bioluminescence imaging have been largely restricted to use in preclinical models. Reasons for the limited clinical translation of optical modalities lie in the inherent limitations imposed by high rates of scattering and poor tissue penetration at the human scale. Each of these limitations leads to increased difficulty in providing the quantitative analysis of data required for practical applications in the clinic. As a consequence of these in vivo limitations, recent advances in the field of optical imaging have focused on developing methods for imaging microscopic events at the cellular and molecular level.

Endoscopy and surgery could benefit from the translation of optical imaging techniques to visualize tumor lesions or metastatic involvement intraoperatively and thereby provide real-time information to guide surgical resection (3). However, at present there are no clinically approved targeted probes for use with targeted fluorescence-reflectance imaging or fluorescence-mediated tomography. Further technical and theoretic challenges mean that to date, the research into developing hybrid systems that combine nuclear and anatomic methods with optical imaging cameras is limited $(1,4)$. Currently intraoperative methods to 
detect radionuclides are limited by the use of hand-held probes that do not provide any spatial information, whereas pure optical approaches are limited by the lack of clinically approved targeted agents. Because 2-dimensional imaging would require large, expensive, and bulky equipment unsuitable for an operating suite, no method is currently available to use the multiplicity of approved radiotracers in the clinic.

The emission of a continuum of ultraviolet and visible light from the decay of certain radionuclides in the condensed phase (now known as the Cerenkov effect) was first observed in 1926 and was characterized by Pavel A. Cerenkov in 1934 (5). Later, in 1958 - and along with his colleagues Ilya Frank and Igor Tamm-Cerenkov was awarded the Nobel Prize in Physics, "for the discovery and the interpretation of the Cherenkov effect." Cerenkov radiation arises when charged particles, such as a $\beta-\left(\beta^{-}\right.$ or $\beta^{+}$) or an $\alpha$-particle, travel through an optically transparent, insulating material with a velocity that exceeds the speed of light, $c$, in the given medium (6). The Cerenkov effect is analogous to the sonic boom that occurs when a macroscopic object such as a jet plane or a whip exceeds the speed of sound in air. As the charged particle travels through the medium, it loses kinetic energy by polarizing the electrons of the insulator (typically water). These polarized molecules then relax back to equilibrium through the emission of ultraviolet and visible light, and when the speed of the charged particle exceeds $c$, constructive interference occurs, giving the observed Cerenkov radiation $(6,7)$.

Although the use of Cerenkov radiation for scintillation counting has been described $(8-11)$, the use of inherent light emission of radionuclides for in vivo imaging is a new concept (12). In a recent paper, Robertson et al. were the first to characterize the use of Cerenkov radiation for the optical imaging of ${ }^{18} \mathrm{~F}$-labeled radiotracers in vivo (13). Further work by Cho et al. (14) and Spinelli et al. (15) verified the origins of visible light emission and paved the way for the development of Cerenkov luminescence imaging (CLI) as a novel in vivo imaging tool.

In this work, we provide further validation of the use of Cerenkov radiation from a much larger range of radionuclides including the positron emitters ${ }^{18} \mathrm{~F},{ }^{64} \mathrm{Cu},{ }^{89} \mathrm{Zr}$, and ${ }^{124} \mathrm{I}$; $\beta$-emitter ${ }^{131} \mathrm{I}$; and $\alpha$-particle emitter ${ }^{225} \mathrm{Ac}$. We report in vitro phantom studies that compare the relative intensity of the optical emission observed from these radionuclides and demonstrate the linear correlation between the observed light output and the measured PET signal. In addition, we also report the feasibility of using CLI for both the qualitative and the quantitative assessment of radiopharmaceutical uptake in tumors in vivo. Uptake of the novel monoclonal antibody ( $\mathrm{mAb}$ )-based radiopharmaceutical ${ }^{89} \mathrm{Zr}$-desferrioxamine B [DFO]-J591 for in vivo immunoimaging of prostate-specific membrane antigen (PSMA) expression in a clinically relevant model of prostate cancer was observed by standard immuno-PET and acute biodistribution studies. The results of these studies are correlated with the tumor uptake observed by CLI. These investigations reveal that optical imaging of Cerenkov radiation shows excellent promise as a potential new in vivo imaging modality for the rapid, low-cost, highthroughput screening of radiopharmaceuticals.

\section{MATERIALS AND METHODS}

Full details of all methods and equipment used are presented in the supplemental materials (available online only at http:// jnm.snmjournals.org).

\section{Radionuclides}

The radionuclides ${ }^{18} \mathrm{~F},{ }^{89} \mathrm{Zr}$, and ${ }^{124} \mathrm{I}$ were produced in high radiochemical and radionuclidic purity via the ${ }^{18} \mathrm{O}-\mathrm{H}_{2} \mathrm{O}(p, n)^{18} \mathrm{~F}$, ${ }^{89} \mathrm{Y}(p, n){ }^{89} \mathrm{Zr}$, and ${ }^{124} \mathrm{TeO}_{2}(p, n){ }^{124} \mathrm{I}$ transmutation reactions on an Ebco TR19/9 variable beam-energy cyclotron (Ebco Industries Inc.) in accordance with previously reported methods (16-20). ${ }^{64} \mathrm{Cu}$ was supplied by the Washington University School of Medicine (21). ${ }^{131}$ I was purchased as a ${ }^{131} \mathrm{I}-\mathrm{NaI}$ (aqueous) solution from MDS Nordion. ${ }^{225}$ Ac was provided as a generous gift from Dr. Michael R. McDevitt and was obtained as a carrier-free product from elution of a ${ }^{229} \mathrm{Th}$ generator system (Oak Ridge National Laboratory) (22). For mAb radiolabeling studies, the ${ }^{89} \mathrm{Zr}$-oxalate reagent was isolated in high radionuclidic and radiochemical purity greater than $99.9 \%$, with an effective specific activity of $195-497 \mathrm{MBq} / \mu \mathrm{g}(5.28-13.43 \mathrm{mCi} / \mu \mathrm{g})(18)$.

\section{Phantom Studies}

For each radionuclide studied, phantoms of varying activity concentration (activity of $0-8.14 \mathrm{MBq}[0-220 \mu \mathrm{Ci}]$ in $200 \mu \mathrm{L}$ of water, for a concentration of $0-40.7 \mathrm{kBq} / \mu \mathrm{L}[0-1.1 \mu \mathrm{Ci} / \mu \mathrm{L}])$ and composed of 6 transparent plastic Eppendorf tubes were prepared by 5 serial 1:2 dilutions of a known amount of activity in deionized water. Phantoms were imaged at various times appropriate to the half-life $\left(t_{1 / 2}\right)$ of the radionuclide under investigation using the Xenogen Ivis 200 device (Caliper Life Sciences). Where possible, PET images of the phantoms were also recorded at the same time points using a microPET Focus 120 scanner (Concorde Microsystems) (23).

\section{Antibody Conjugation and ${ }^{89} \mathrm{Zr}$ Radiolabeling}

The $\mathrm{IgG}_{1} \mathrm{mAb}$ J591 was conjugated to the tris-hydroxamate, hexadentate chelate DFO (Calbiochem) using a 6-step procedure modified (24) from that described by Verel et al. (19) (supplemental materials).

\section{Xenograft Models}

All animal experiments were conducted in compliance with Institutional Animal Care and Use Committee guidelines and the Guide for the Care and Use of Laboratory Animals (25). All animal procedures were performed under anesthesia by inhalation of a 1\%-2\% isoflurane (Baxter Healthcare)-oxygen mixture. Full details are presented in the supplemental materials.

\section{PET}

PET experiments were conducted on a microPET Focus 120 scanner (23). PET images of the in vitro phantoms were recorded using the same methods and instrument parameters as described for the small-animal immuno-PET studies.

Mice were administered ${ }^{89} \mathrm{Zr}$-DFO-J591 formulations (10.911.3 MBq [295-305 $\mu \mathrm{Ci}$ ], 60-62 $\mu \mathrm{g}$ of mAb, in $200 \mu \mathrm{L}$ of sterile saline for injection) via retroorbital injection. Approximately 
5 min before PET images were recorded, mice were anesthetized by inhalation of a $1 \%-2 \%$ isoflurane-oxygen gas mixture and placed on the scanner bed. PET images were recorded at various times between 24 and $96 \mathrm{~h}$ after injection. List-mode data were acquired for between 10 and 30 min using a $\gamma$-ray energy window of $350-750 \mathrm{keV}$ and a coincidence timing window of $6 \mathrm{~ns}$ (the supplemental materials provide additional details).

\section{Optical Imaging}

Optical images were acquired using the Xenogen Ivis 200 optical imager. Cerenkov radiation was detected from each phantom containing various activities of the same radionuclide using the bioluminescence setting (integration time, 10, 20, 30, 40, 50, and $60 \mathrm{~s}$; f/stop, 1; binning, medium; field of view, B), with no light interference from the excitation lamp. Spectral analysis was obtained by measuring optical images either with or without the use of a narrow band filter $(560,580,600,620,640$, and 680, or open filter) of $20 \mathrm{~nm}$ in full width at half maximum (the supplemental materials provide additional details).

\section{Acute Biodistribution Studies}

Acute in vivo biodistribution studies were conducted at the end of the optical imaging and immuno-PET to validate the uptake and localization of ${ }^{89} \mathrm{Zr}$-DFO-J591 observed in mice bearing dual subcutaneous LNCaP $\left(50-250 \mathrm{~mm}^{3}\right)$ tumors $(n=3)$. Full details are presented in the supplemental materials.

\section{Statistical Analysis}

Data were analyzed using the unpaired, 2-tailed Student $t$ test. Differences at the $95 \%$ confidence level $(P<0.05)$ were considered to be statistically significant.

\section{RESULTS}

\section{Phantom Studies}

The ability to visualize and quantify the Cerenkov radiation emitted from a range of positron, $\beta$-, and $\alpha$-emitting radionuclides was first investigated using in vitro phantom studies. Standard solutions of decreasing activity concentration (ranging from 0 to $40.7 \mathrm{kBq} / \mu \mathrm{L}$ ) of each radionuclide in water $(200 \mu \mathrm{L})$ were prepared by $1: 2$ serial dilution and subjected to optical imaging at various times appropriate for the $t_{1 / 2}$ of the nuclide under investigation. For the positron-emitting radionuclides, the phantoms were also imaged using PET. Typical optical and PET images of the phantom are shown in Figures $1 \mathrm{~A}$ and $1 \mathrm{~B}$, respectively. The phantom images recorded with the other radionuclides ${ }^{18} \mathrm{~F},{ }^{64} \mathrm{Cu},{ }^{89} \mathrm{Zr},{ }^{124} \mathrm{I},{ }^{131} \mathrm{I}$, and ${ }^{225} \mathrm{Ac}$ were qualitatively equivalent. However, for reasons of clarity and consistency with the in vivo studies, our analysis and discussion focus on ${ }^{89} \mathrm{Zr}$.

The data acquired from the optical and PET phantom studies were also quantitatively analyzed. Figure 2A shows a plot of the average (background-corrected) radiance $(\mathrm{p} / \mathrm{s} /$ $\left.\mathrm{cm}^{2} / \mathrm{sr}\right)$ versus the ${ }^{89} \mathrm{Zr}$ activity concentration $(\mathrm{kBq} / \mu \mathrm{L})$. Background correction was applied by subtracting the radiance measured from a region of interest (ROI) of the optical images drawn over the sample containing no activity. Linear regression analysis reveals a strong, positive correlation between the light emission intensity and activity
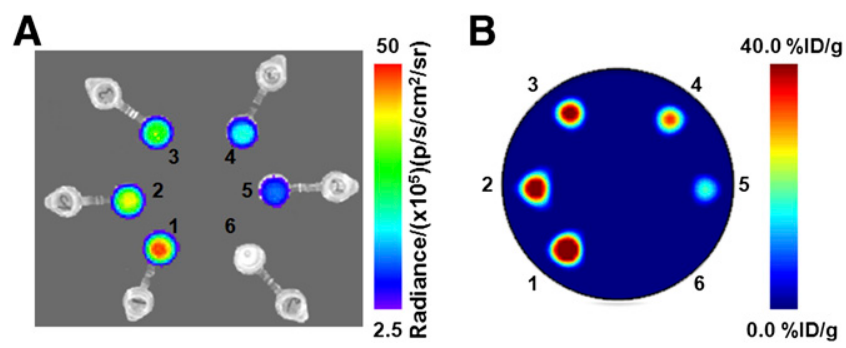

FIGURE 1. Phantom images recorded using optical (CLI) imaging (A) and PET (B) of 6 samples of ${ }^{89} \mathrm{Zr}$ activity in water. At time $0 \mathrm{~h}$, the Eppendorf tubes labeled 1-6 corresponded to activity concentrations of $40.3,32.6,27.4,20.4,13.3$, and $0.00 \mathrm{kBq} / \mu \mathrm{L}$. Optical images were recorded using integration time of $30 \mathrm{~s}$ and f/stop of 1.

concentration, with a correlation coefficient of $R=0.98$. Figure 2B displays a plot of the normalized radiance versus time, $t / \mathrm{h}$, for the first two ${ }^{89} \mathrm{Zr}$ samples (Fig. 1, tubes 1 and 2). Exponential fitting of the data using the standard equation for first-order radioactive decay gave an excellent correlation $(R=0.98)$, with a calculated $\mathrm{t}_{1 / 2}\left({ }^{89} \mathrm{Zr}\right.$ radiance) equal to $79.1 \pm 4.8 \mathrm{~h}$. This experimentally measured $\mathrm{t}_{1 / 2}$ is consistent with the known rate of decay of ${ }^{89} \mathrm{Zr}\left(\mathrm{t}_{1 / 2}=\right.$ $78.41 \mathrm{~h}$ ), which provides additional evidence that the source of the radiation arises directly from the radionuclide decay (16). Furthermore, the optical emission profile observed for ${ }^{18} \mathrm{~F},{ }^{64} \mathrm{Cu},{ }^{89} \mathrm{Zr},{ }^{124} \mathrm{I},{ }^{131} \mathrm{I}$, and ${ }^{225} \mathrm{Ac}$ was found to be the same as previously reported for ${ }^{18} \mathrm{~F}$ and is consistent with Cerenkov radiation (13-15).

To assess the potential for using the observed optical emission of radionuclides for quantitative analysis of the images, the relationship between optical ROI and PET volume of interest was examined. Figure $2 \mathrm{C}$ shows a plot of the average radiance $\left(\mathrm{p} / \mathrm{s} / \mathrm{cm}^{2} / \mathrm{sr}\right)$ versus the mean activity measured by PET (presented in units of percentage injected dose per gram [\% ID/g], which are commonly used for in vivo analysis of radiotracer uptake). A linear relationship $(R=0.98)$ was observed between the optical and PET signal intensities, suggesting that CLI is, in principle, quantitative. The relationship presented here represents an in vitro system for which the effects of depth- and mediumdependent scattering are expected to be minimized. For in vivo imaging, tissue penetration and scattering of light in the ultraviolet and visible regions of the spectrum will complicate the quantification of Cerenkov emission data.

To assess the relative utility of different radionuclides for use in CLI, we investigated the relative light output from each of the available radionuclides. Figure 3 shows a plot of the ratio of the background-corrected average radiance to the activity concentration (in units of $\left[\mathrm{p} / \mathrm{s} / \mathrm{cm}^{2} / \mathrm{sr}\right] /[\mathrm{kBq} /$ $\mu \mathrm{L}])$ versus the radionuclide. To facilitate comparison, the positron-emitting radionuclides have been arranged in order of increasing mean $\beta^{+}$kinetic energy/keV. The relationship between the number of positrons emitted in a given energy range and the theoretic number of photons produced in 

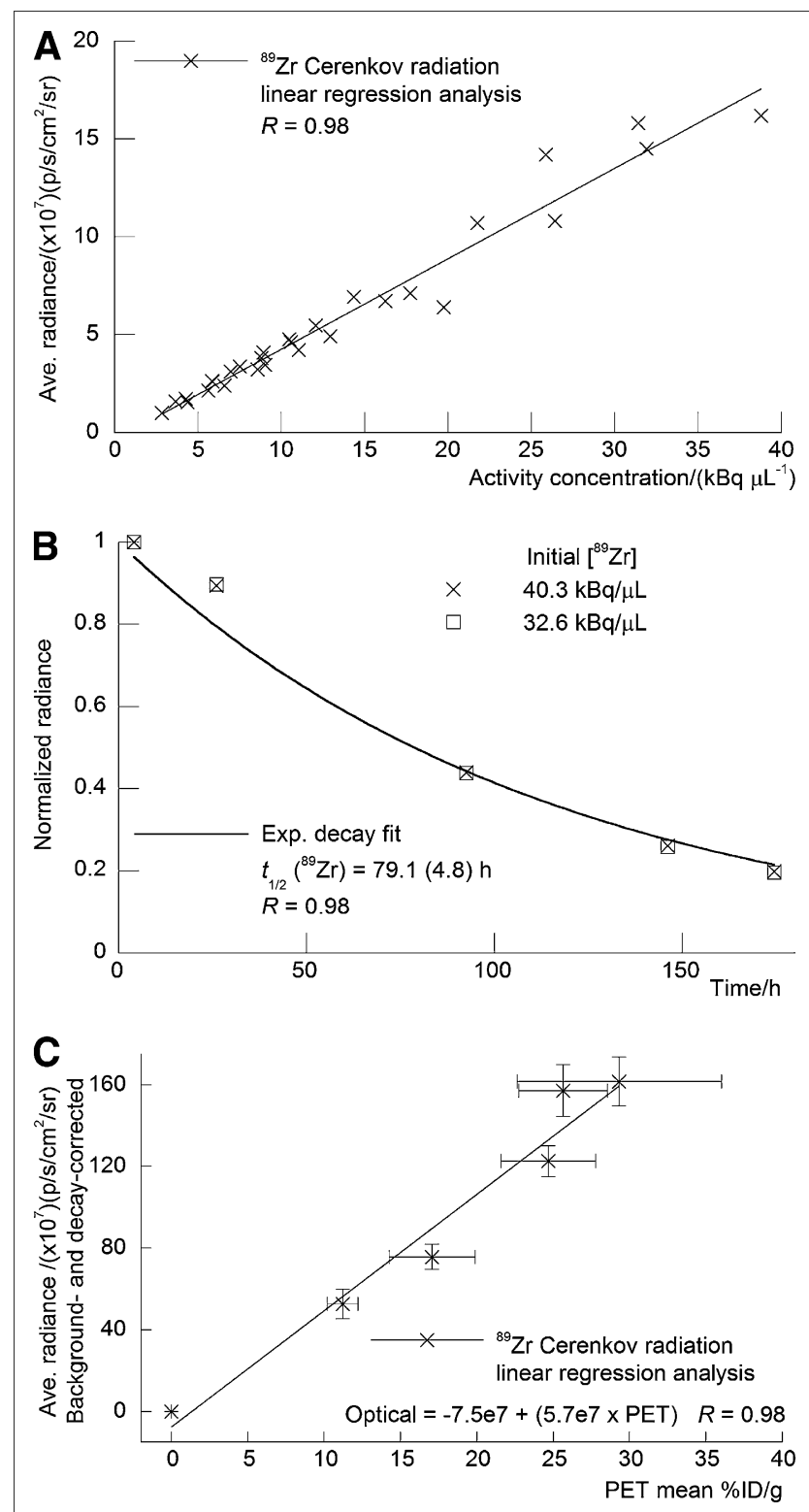

FIGURE 2. Quantitative analysis of phantom studies. Positive correlation observed between measured average radiance (background-corrected in units of $\mathrm{p} / \mathrm{s} / \mathrm{cm}^{2} / \mathrm{sr}$ ) and ${ }^{89} \mathrm{Zr}$ activity concentration $(\mathrm{kBq} / \mu \mathrm{L})(\mathrm{A})$, rate of decay observed in normalized radiance vs. time/h $(B)$, and linear correlation observed between average radiance (background and decay-corrected in units of $\mathrm{p} / \mathrm{s} / \mathrm{cm}^{2} / \mathrm{sr}$ ) vs. mean PET signal intensity (measured in units of \%ID $/ \mathrm{g}$, commonly used for quantification of in vivo PET studies) (C). Ave. = average; Exp. = exponential.

a medium of known refractive index is well established $(6,15,26)$. Despite the fact that the threshold for producing coherent Cerenkov radiation in water is $263 \mathrm{keV}(6,7),{ }^{18} \mathrm{~F}$ with a mean $\beta^{+}$kinetic energy of $249.8 \mathrm{keV}$ and positron yield of $\mathrm{I}_{\beta}{ }^{+}=100 \%$ gives a measured radiance for light output comparable to the higher-energy emitter ${ }^{89} \mathrm{Zr}\left(\mathrm{E}_{\beta}{ }^{+}=\right.$ $395.5 \mathrm{keV} ; \mathrm{I}_{\beta}{ }^{+}=22.7 \%$ ). As expected for the positron-

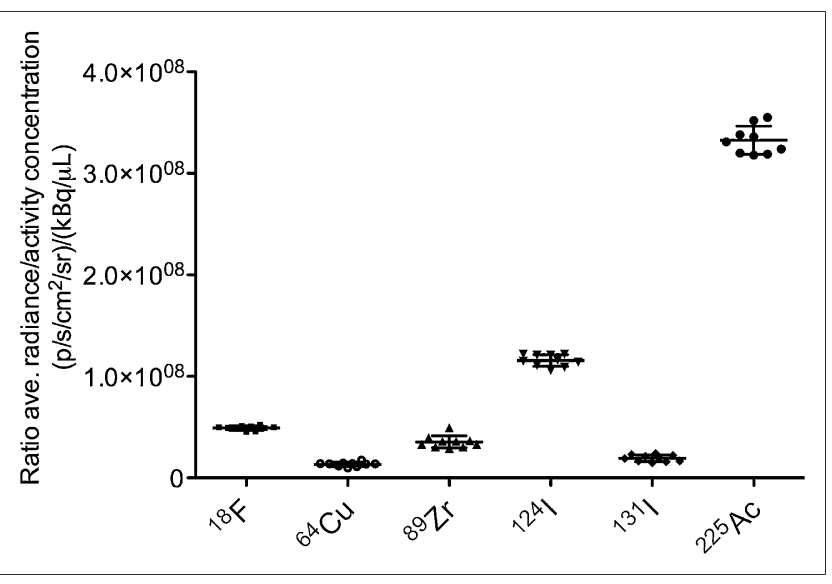

FIGURE 3. Plot of ratio of average radiance $\left(p / s / \mathrm{cm}^{2} / \mathrm{sr}\right) /$ activity concentration $(\mu \mathrm{Ci} / \mu \mathrm{L})$ vs. radionuclide. Positronemitting radionuclides are arranged in order of increasing average $\beta^{+}$kinetic energy $\left({ }^{18} \mathrm{~F}: \mathrm{E}_{\beta}{ }^{+}=249.8 \mathrm{keV}\left[\mathrm{I}_{\beta}{ }^{+}=\right.\right.$ 100\%]; ${ }^{64} \mathrm{Cu}: \mathrm{E}_{\beta}{ }^{+}=278.2 \mathrm{keV}\left[\mathrm{I}_{\beta}{ }^{+}=17.6 \%\right] ;{ }^{89} \mathrm{Zr}: \mathrm{E}_{\beta}{ }^{+}=$ $395.5 \mathrm{keV}\left[\mathrm{I}_{\beta}{ }^{+}=22.7 \%\right]$; $\left.{ }^{124} \mathrm{I}: \mathrm{E}_{\beta}{ }^{+}=820 \mathrm{keV}\left[\mathrm{I}_{\beta}{ }^{+}=22.7 \%\right]\right)$. For ${ }^{131} \mathrm{I}, \mathrm{E}_{\beta}{ }^{-}=181.9 \mathrm{keV}\left(\mathrm{I}_{\beta}{ }^{-} 100 \%\right) .{ }^{225} \mathrm{Ac}$ decays by $100 \%$ $\alpha$-particle emission with $E_{\alpha}$ in range of $5,021-5,830 \mathrm{keV}(16)$. Ave. $=$ average.

emitting radionuclides, the higher-energy decay of ${ }^{124} \mathrm{I}$ was found to give the most intense Cerenkov radiation.

${ }^{225} \mathrm{Ac}$ was found to give the most intense optical radiation (Fig. 3). ${ }^{225} \mathrm{Ac}$ is a pure $(100 \%) \alpha$-emitter, and its decay releases $\alpha$-particles with energies in the range of 5,021 to $5,830 \mathrm{keV}$. This energy range is considerably higher than the energy of the most energetic positrons studied in this work $\left({ }^{124} \mathrm{I}: \mathrm{E}_{\beta}{ }^{+}[\right.$maximum] $=2,137.6 \mathrm{keV})$. However, because of their relatively large size (mass), $\alpha$-particles are known to travel at velocities below the threshold for Cerenkov radiation (27). The origins of the optical emissions observed from ${ }^{225} \mathrm{Ac}$ remain uncertain, although it is possible that the optical emissions originate from a series of short-lived, $\beta$-emitting daughter nuclides including ${ }^{213} \mathrm{Bi}\left(\mathrm{t}_{1 / 2}=45.59 \mathrm{~min}, \mathrm{E}_{\beta}{ }^{-}[\right.$mean $]=435 \mathrm{keV}$, $\left.\mathrm{I}_{\beta}{ }^{-}=97.8 \%\right),{ }^{209} \mathrm{~Pb}\left(\mathrm{t}_{1 / 2}=3.253 \mathrm{~h}, \mathrm{E}_{\beta}{ }^{-}[\right.$mean $]=197.5$ $\left.\mathrm{keV}, \mathrm{I}_{\beta}{ }^{-}=100 \%\right)$, and ${ }^{209} \mathrm{Tl}\left(\mathrm{t}_{1 / 2}=2.20 \mathrm{~min}, \mathrm{E}_{\beta}{ }^{-}[\right.$mean $]=$ $\left.656 \mathrm{keV}, \mathrm{I}_{\beta}{ }^{-}=100 \%\right)(28)$.

The decay of ${ }^{225} \mathrm{Ac}$ cannot be measured by current nuclear imaging modalities. Validation of the use of ${ }^{225} \mathrm{Ac}$ for optical imaging provides an example of the potential applications of dedicated CLI devices. A range of ${ }^{225} \mathrm{Ac}$-labeled mAb-based agents has been developed (28-31). In an ongoing phase I clinical trial between Memorial Sloan-Kettering Cancer Center (MSKCC) and the National Cancer Institute, ${ }^{225} \mathrm{Ac}$ conjugated to the humanized anti-CD33 mAb ${ }^{225} \mathrm{Ac}-\mathrm{HuM} 195$ is under evaluation as a radioimmunotherapeutic agent for targeted therapy of leukemia and myelodysplastic syndrome (clinical trial NCT00672165). The ability to measure the tumor targeting of ${ }^{225} \mathrm{Ac}$-labeled $\mathrm{mAbs}$ and potentially estimate 
in vivo dosimetry using optical imaging would represent a fundamental advance in imaging science.

\section{Radiochemistry}

To assess the potential of optical CLI of tumors in vivo, we developed the ${ }^{89} \mathrm{Zr}$-labeled mAb ${ }^{89} \mathrm{Zr}$-DFO-J591 (3236). Several examples of the radiolabeling, characterization, and use of ${ }^{89} \mathrm{Zr}-\mathrm{DFO}-\mathrm{mAbs}$ for immuno-PET of various cancers have been reported (24,37-41).

In these studies, the humanized mAb J591, which binds to an extracellular epitope of PSMA expressed in most prostate cancer cell lines, was functionalized with the trishydroxamate chelate DFO using bioconjugation methods modified (24) from the pioneering work of Verel et al. (19). Full details of the $\mathrm{mAb}$ conjugation, identification, and ${ }^{89} \mathrm{Zr}$ radiolabeling and in vitro and in vivo characterization of ${ }^{89} \mathrm{Zr}$-DFO-J591 will be reported elsewhere. The final radiochemical yield of the purified ${ }^{89} \mathrm{Zr}$-DFO-J591 was $67 \%$, and the product was formulated in $0.9 \%$ sterile saline with a radiochemical purity greater than $99 \%$ and a specific activity of $165.0 \mathrm{MBq} / \mathrm{mg}(4.47 \mathrm{mCi} / \mathrm{mg})$ of $\mathrm{mAb}$ (Supplemental Figs. 1 and 2).

\section{In Vivo Studies}

The site-specific localization of ${ }^{89} \mathrm{Zr}$-DFO-J591 in subcutaneous human xenograft LNCaP (PSMA-positive) tumors was used to assess the ability of optical CLI and immuno-PET to provide both qualitative and quantitative data on the biodistribution of a radiotracer in vivo. Temporal images of ${ }^{89} \mathrm{Zr}-\mathrm{DFO}-J 591 \quad(10.9-11.3 \mathrm{MBq}$ [295-305 $\mu \mathrm{Ci}$ ], $60-62 \mu \mathrm{g}$ of mAb in $200 \mu \mathrm{L}$ of sterile saline) tumor uptake recorded between 24 and $96 \mathrm{~h}$ after retroorbital administration using CLI and immuno-PET are presented in Figures $4 \mathrm{~A}$ and $4 \mathrm{~B}$, respectively. The mice were shaved to reduce signal scattering before imaging at $24 \mathrm{~h}$ (15). The images in Figure 4A demonstrate that optical imaging of Cerenkov radiation derived from the decay of an administered radiotracer can be achieved in vivo. In these studies each mouse was inoculated on both the right and the left flanks with LNCaP cells. Tumors grew on both flanks, but in each animal the growth of 1 tumor exceeded that of the other by between 3- and 4-fold, providing 2 different datasets classified as either small or large LNCaP tumors. Figure 4A also shows that differential uptake between the small and large tumors can be discerned by

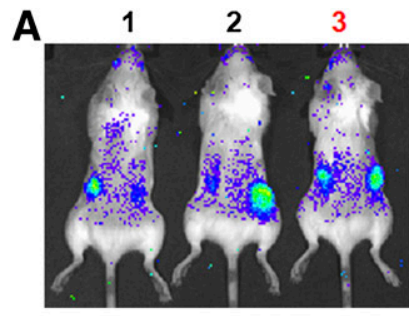

$24 \mathrm{~h}$

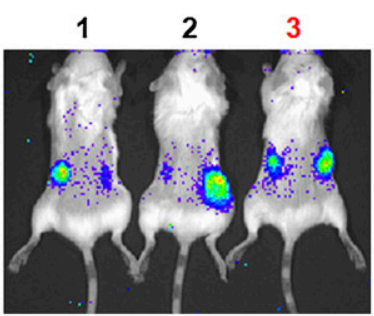

$48 \mathrm{~h}$

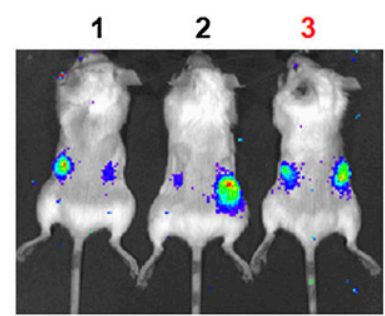

$72 \mathrm{~h}$

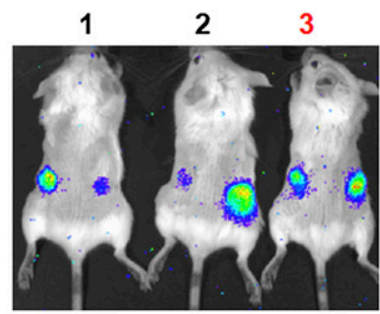

$96 \mathrm{~h}$

20 100

Radiance $/\left(\times 10^{3}\right)\left(\mathrm{p} / \mathrm{s} / \mathrm{cm}^{2} / \mathrm{sr}\right)$

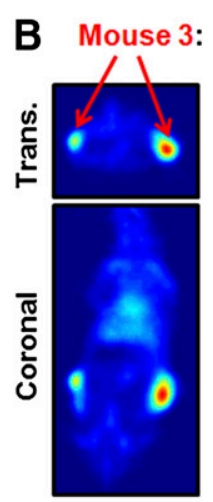

$24 \mathrm{~h}$

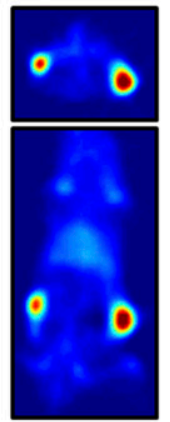

$48 \mathrm{~h}$

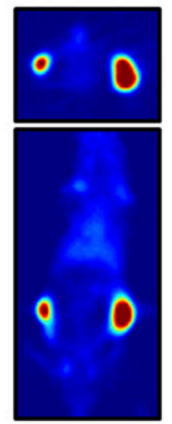

$72 \mathrm{~h}$

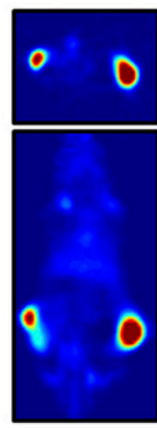

$96 \mathrm{~h}$

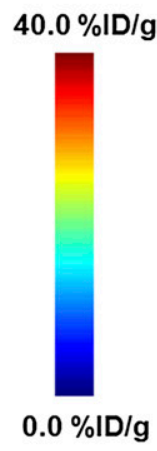

C
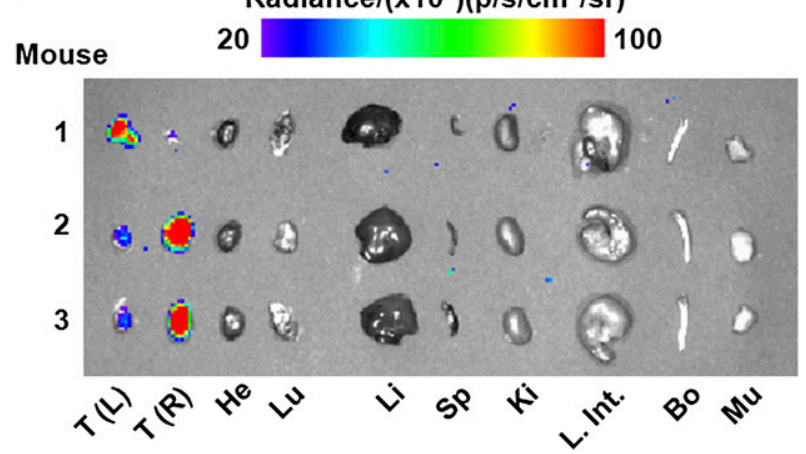

FIGURE 4. Temporal images of ${ }^{89} \mathrm{Zr}$-DFO-J591 uptake (10.9-11.3 MBq [295-305 $\mu \mathrm{Ci}$ ], $60-62 \mu \mathrm{g}$ of mAb, in $200 \mu \mathrm{L}$ of $0.9 \%$ sterile saline) recorded in dual subcutaneous LNCaP (PSMA-positive) tumor-bearing severe combined immune deficient mice between 24 and $96 \mathrm{~h}$ after administration. (A) Signal observed in optical spectrum from in vivo CLI of ${ }^{89} \mathrm{Zr}$-DFO-J591 tumor uptake in 3 mice. (B) Corresponding coronal and transverse immuno-PET images recorded for mouse 3 . (C) Optical image recorded of organs after acute ex vivo biodistribution at $96 \mathrm{~h}$. Transverse and coronal planar immuno-PET images intersect center of tumors. Upper and lower thresholds of CLI and immuno-PET images in A-C have been adjusted for visual clarity, as indicated by scale bars. Trans. = transverse; +ve = positive; $\mathrm{T}(\mathrm{L})=$ left tumor; $\mathrm{T}(\mathrm{R})=$ right tumor; $\mathrm{He}=$ heart; $\mathrm{Lu}=$ lungs; $\mathrm{Li}=$ liver; $\mathrm{Sp}=$ spleen; $\mathrm{Ki}=$ kidneys; $\mathrm{L}$. Int. = large intestine; Bo = bone; $\mathrm{Mu}=$ muscle. 
optical CLI. This result is, to the best of our knowledge, the first demonstration of specific tumor imaging using the inherent Cerenkov radiation emitted from a metallolabeled immunoconjugate. Furthermore, qualitative analysis of the CLI pictures indicates that higher ${ }^{89} \mathrm{Zr}$-DFO-J591 background activity is present at $24 \mathrm{~h}$, with lower uptake in the tumors. Between 24 and $96 \mathrm{~h}$, tumor uptake of ${ }^{89} \mathrm{Zr}$-DFOJ591 continues to increase and activity in the background shows a concordant decrease (as deduced by observation of ROIs located over the mice but remote from the tumor locations).

The corresponding ${ }^{89} \mathrm{Zr}$-DFO-J591 temporal immunoPET images of a representative animal (mouse 3) recorded at the same times as the CLI pictures are shown in Figure 4B. Transverse and coronal slices are taken through the center of the tumors. For mouse 3 , the $\mathrm{LNCaP}$ tumor located in the right flank was approximately 3 times larger in volume $\left(250 \mathrm{~mm}^{3}\right)$ than the tumor in the left flank $\left(80 \mathrm{~mm}^{3}\right)$. The first observation is that ${ }^{89} \mathrm{Zr}$-DFO-J591 provides excellent contrast for the delineation of tumorversus-background tissue uptake using immuno-PET. Furthermore, facile distinction between the radiotracer uptake and accumulation in the small and large tumors was observed, and tumor uptake continued to increase during the full time course of the immuno-PET experiments. These immuno-PET studies provide a reference point for the interpretation of the CLI data and confirm that the observed qualitative increase in optical intensity of the tumor ROIs over time is due to an increase in radiotracer accumulation. Full quantitative analysis is discussed in the next sections.

At the end of the imaging experiment $(96 \mathrm{~h})$, the mice were sacrificed and subjected to both optical imaging of the excised organs and acute ex vivo biodistribution studies to quantify the accumulation of ${ }^{89} \mathrm{Zr}$ radioactivity. An optical image of the excised organs from mice $1-3$ is shown in Figure $4 \mathrm{C}$. The organ image demonstrates that at the same optical emission settings and threshold values as used for the in vivo images, uptake in only the LNCaP tumors was observed. The lack of ${ }^{89} \mathrm{Zr}$ activity in the optical image of the background organs is consistent with the immuno-PET images recorded at $96 \mathrm{~h}$, suggesting excellent clearance of ${ }^{89} \mathrm{Zr}$-DFO-J591 from nontarget tissue.

Results from the acute biodistribution study at $96 \mathrm{~h}$ are presented as a bar chart in Figure 5. The data confirm that high uptake of ${ }^{89} \mathrm{Zr}$-DFO-J591 in PSMA-positive tumors occurs within $96 \mathrm{~h}$ after administration. Differential radiotracer uptake and accumulation were observed between the large and small tumor groups. For the larger LNCaP tumors (average volume, $220 \mathrm{~mm}^{3}$ ), radiotracer uptake reached $72.3 \pm 4.6 \% \mathrm{ID} / \mathrm{g}$. However, for the smaller tumors (average volume, $65 \mathrm{~mm}^{3}$ ), radiotracer uptake was still well above background tissue uptake but reached only $32.0 \pm$ $5.4 \% \mathrm{ID} / \mathrm{g}(P=0.0007)$. The dependence of radiotracer accumulation on tumor size is likely due to enhanced vascularization and the increased number of available PSMA epitopes presented by the well-established tumors.

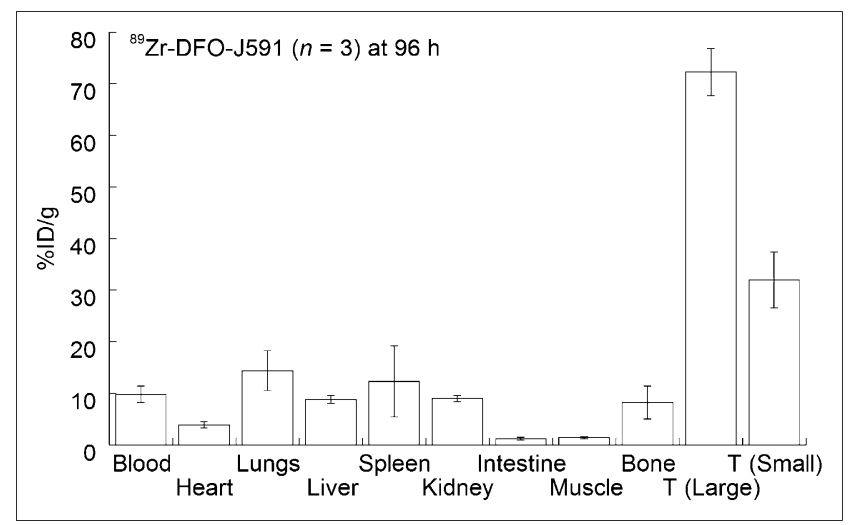

FIGURE 5. Bar chart showing selected tissue biodistribution data $(\% \mathrm{ID} / \mathrm{g})$ for uptake of ${ }^{89} \mathrm{Zr}$-DFO-J591 in male severe combined immune deficient mice at end of optical and immuno-PET experiments (96 $\mathrm{h}$ after injection). $\mathrm{T}=$ tumor.

Overall, these biodistribution data are consistent with the optical and immuno-PET studies.

For an imaging modality to be useful in a clinical setting, it is essential that the data obtained be at least semiquantitative. Figure 6 shows the time-activity curves derived from ROI and volume-of-interest analysis of the in vivo optical and immuno-PET images. The time-activity curves confirm that ${ }^{89} \mathrm{Zr}$-DFO-J591 is efficiently removed from the blood pool and accumulated only to low levels in background tissue. The time-activity curves also show that ${ }^{89} \mathrm{Zr}$-DFO-J591 uptake in the larger tumors increased between 24 and $96 \mathrm{~h}$. For example, the immuno-PET tumor time-activity curve shows an increase in radiotracer accumulation from $16.7 \pm 1.4 \% \mathrm{ID} / \mathrm{g}$ at $24 \mathrm{~h}$ to $45.1 \pm 10.3 \%$ $\mathrm{ID} / \mathrm{g}$ at $96 \mathrm{~h}$, corresponding to a $2.5- \pm 0.8$-fold increase. ROI

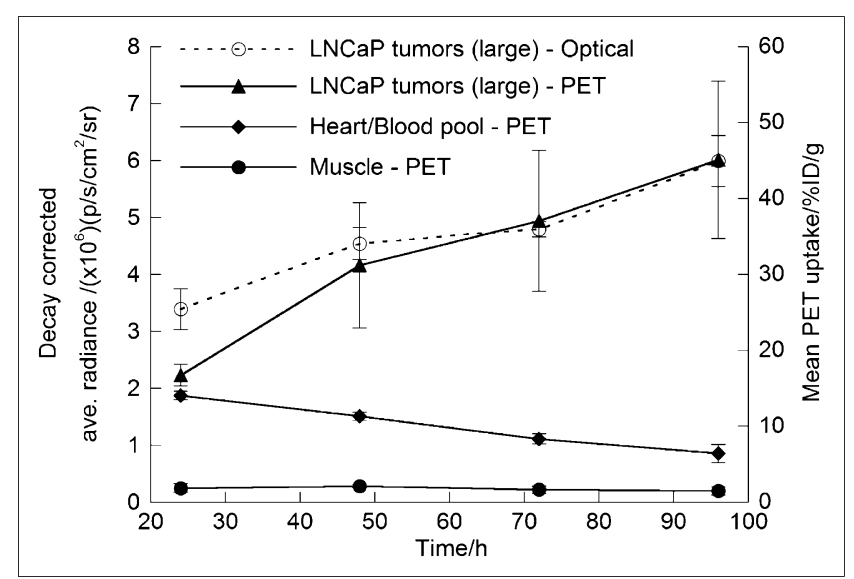

FIGURE 6. Time-activity curves showing ROI and volumeof-interest analysis of CLI and immuno-PET images for ${ }^{89} \mathrm{Zr}$ DFO-J591 uptake in well-established (large) LNCaP tumors. Volume-of-interest analysis of immuno-PET images shows change in ${ }^{89} \mathrm{Zr}$ activity in heart-blood pool and muscle tissue. 
analysis of the optical images also showed the same increase in radiotracer uptake in the larger tumors over time. The measured, decay-corrected, average radiance for the larger tumors increased from $3.4 \pm 0.35$ to $6.0 \pm 0.45 \mathrm{p} / \mathrm{s} / \mathrm{cm}^{2} / \mathrm{sr}$ at 24 and $96 \mathrm{~h}$, respectively. This change in measured tumor radiance corresponds to a 1.8- \pm 0.3 -fold increase over time, which is similar to changes observed from the immuno-PET studies. Indeed, accounting for the relative errors in the 2 measurements, the observed change in CLI intensity for ${ }^{89} \mathrm{Zr}$-DFOJ591 tumor accumulation was the same as for immuno-PET.

\section{DISCUSSION}

In 2009, Robertson et al. reported proof-of-concept studies that validated the use of Cerenkov radiation emissions in the ultraviolet and visible regions of the spectrum for in vivo optical imaging of ${ }^{18} \mathrm{~F}$-radiolabeled compounds (13). This new technique was termed CLI and represents a fundamental advance toward the development of hybrid nuclear-optical tomographic imaging devices. In this work, we explored the potential of imaging Cerenkov radiation with a wider range of radionuclides and provided the first examples of dual optical CLI and PET of in vivo tumor uptake using a metallolabeled antibody.

The in vitro and in vivo imaging studies presented in this work demonstrate not only that the inherent Cerenkov emissions of various clinically relevant radionuclides can be visualized but also that the data obtained correlate with the observed biodistribution of radiotracers. In addition, both qualitative and quantitative interpretation of the CLI data was found to give a strong correlation with immunoPET and biodistribution studies. We anticipate that CLI of administered radiopharmaceuticals has a broad range of potential applications. In particular, the ability to simultaneously measure time-dependent changes in tumor uptake of radiotracers in multiple different tumor models or chemotherapeutic treatment regimes means that optical CLI offers the potential to conduct rapid, low-cost, highthroughput screening of novel radiotracers in vivo. In addition, intraoperative imaging of Cerenkov radiation is feasible with a highly sensitive camera in a dark room, the use of which can be achieved much more easily than with expensive nuclear medicine equipment. Notably, no additional development of optical agents is required because CLI can take advantage of many approved radiopharmaceuticals. Therefore, the combination of endoscopic surgical methods with optical Cerenkov imaging has the potential to be used directly in the clinic for intraoperative visualization of tumor lesions and margins or metastatic involvement for Cerenkov radiation-guided surgery. Further studies using hybrid nuclear-optical imaging of radiopharmaceuticals are under way at MSKCC.

\section{CONCLUSION}

Basic characterization of the inherent Cerenkov radiation emission from a range of positron-, $\beta-$, and $\alpha$-emitting radionuclides commonly used as imaging and therapeutic isotopes in nuclear medicine is reported. Phantom studies confirm that the broad-spectrum optical emissions arise because of Cerenkov radiation in the condensed phase. The measured radiance was found to give a strong correlation with the known activity concentration, and the signal intensity was found to correlate with the known $t_{1 / 2}$ of the nuclides under investigation. In addition, in vitro studies comparing the optical signal intensity with that observed from the PET images of the positron-emitting nuclides revealed a linear correlation, indicating that CLI has the potential to provide quantitative data analysis of radiotracers.

In vivo studies looking at the uptake of ${ }^{89} \mathrm{Zr}$-DFO-J591 in PSMA-positive LNCaP prostate tumors demonstrated that for the first time, to our knowledge, Cerenkov radiation emission can be used to quantify the tumor-specific uptake of a novel targeted, metallolabeled tracer. Furthermore, time-activity curves revealed that the intensity of the observed optical signal correlated with the quantitative immuno-PET and acute biodistribution studies. These results pave the way for further use and development of CLI as a novel optical imaging modality for the rapid, costeffective, high-throughput screening of radiopharmaceuticals. Studies aimed at using the Cerenkov radiation emitted by various radionuclides in the development of activatable reporter system probes are under way at MSKCC.

\section{ACKNOWLEDGMENTS}

We thank Drs. NagaVaraKishore Pillarsetty, and Pat Zanzonico for informative discussions; Valerie M. Longo for assistance with the imaging experiments; Thomas Ku and Vadim Divilov for advice on in vitro experiments; and Bradley Beattie for assistance with the optical imaging. We thank Dr. Neil H. Bander (Weill Medical College of Cornell University) for the generous gift of J591. We also thank the staff of the Radiochemistry/Cyclotron Core at MSKCC. This study was funded in part by the Office of Science (BER), U.S. Department of Energy (award DE-SC0002456); the Geoffrey Beene Cancer Research Center of Memorial Sloan-Kettering Cancer Center; and the NIH (P30 CA008748-44S5 and R25T CA046945). Technical services provided by the MSKCC Small-Animal Imaging Core Facility were supported in part by NIH grants R24 CA83084 and P30 CA08748.

\section{REFERENCES}

1. Weissleder R, Pittet MJ. Imaging in the era of molecular oncology. Nature. 2008; 452:580-589.

2. Pichler BJ, Kolb A, Nagele T, Schlemmer H-P. PET/MRI: paving the way for the next generation of clinical multimodality imaging applications. J Nucl Med. 2010;51:333-336.

3. Kirsch DG, Dinulescu DM, Miller JB, et al. A spatially and temporally restricted mouse model of soft tissue sarcoma. Nat Med. 2007;13:992-997.

4. Grimm J, Kirsch DG, Windsor SD, et al. Use of gene expression profiling to direct in vivo molecular imaging of lung cancer. Proc Natl Acad Sci. 2005;102: 14404-14409.

5. Cerenkov PA. Visible emission of clean liquids by action of $\gamma$-radiation. $C R$ Dokl Akad Nauk SSSR. 1934;2:451-454. 
6. Jelley JV. Cerenkov radiation and its applications. Br J Appl Phys. 1955;6: 227-232.

7. Ross HH. Measurement of $\beta$-emitting nuclides using Cerenkov radiation. Anal Chem. 1969;41:1260-1265.

8. Plesums J, Bunch WH. Measurement of phosphorus following ${ }^{32} \mathrm{P}$ Cerenkov counting. Anal Biochem. 1971;42:360-362.

9. Simonnet F, Combe J, Simonnet G. Detection of ${ }^{32} \mathrm{P}$ scintillating plastic vials. Appl Radiat Isot. 1987;38:311-312.

10. Hansen BS. Improved method for assaying pyrophosphate exchange measuring Cerenkov radiation. Anal Biochem. 1980;109:12-17.

11. Berger SL. The use of Cerenkov radiation for monitoring reactions performed in minute volumes: examples from recombinant DNA technology. Anal Biochem. 1984;136:515-519.

12. Miyata M, Tomita H, Watanabe K, Kawarabayshi J, Iguchi T. Development of TOF-PET using Cherenkov radiation. J Nucl Sci Technol. 2006;43:339-343.

13. Robertson R, Germanos MS, Li C, Mitchell GS, Cherry SR, Silva MD. Optical imaging of Cerenkov light generation from positron-emitting radiotracers. Phys Med Biol. 2009;54:N355-N365.

14. Cho JS, Taschereau R, Olma S, et al. Cerenkov radiation imaging as a method for quantitative measurements of beta particles in a microfluidic chip. Phys Med Biol. 2009;54:6757-6771.

15. Spinelli AE, D'Ambrosio D, Calderan L, Marengo M, Sbarbati A, Boschi F. Cerenkov radiation allows in vivo optical imaging of positron emitting radiotracers. Phys Med Biol. 2010;55:483-495.

16. Holland JP, Williamson MJ, Lewis JS. Unconventional nuclides for radiopharmaceuticals. Mol Imaging. 2010;9:1-20.

17. Welch MJ, Redvanly CS, Editors. Handbook of Radiopharmaceuticals: Radiochemistry and Applications. New York, NY: Wiley; 2003.

18. Holland JP, Sheh Y, Lewis JS. Standardized methods for the production of high specific-activity zirconium-89. Nucl Med Biol. 2009;36:729-739.

19. Verel I, Visser GWM, Boellaard R, Stigter-van Walsum M, Snow GB, van Dongen GAMS. ${ }^{89} \mathrm{Zr}$ immuno-PET: comprehensive procedures for the production of ${ }^{89} \mathrm{Zr}$-labeled monoclonal antibodies. J Nucl Med. 2003;44: 1271-1281.

20. Sheh Y, Koziorowski J, Balatoni J, Lom C, Dahl JR, Finn RD. Low energy cyclotron production and chemical separation of "no carrier added" iodine-124 from a reusable, enriched tellurium-124 dioxide/aluminum oxide solid solution target. Radiochim Acta. 2000;88:169-173.

21. McCarthy DW, Shefer RE, Klinkowstein RE, et al. Efficient production of high specific activity ${ }^{64} \mathrm{Cu}$ using a biomedical cyclotron. Nucl Med Biol. 1997;24:3543.

22. Miederer M, Scheinberg DA, McDevitt MR. Realizing the potential of the actinium-225 radionuclide generator in targeted alpha particle therapy applications. Adv Drug Deliv Rev. 2008;60:1371-1382.

23. Kim JS, Lee JS, Im KC, et al. Performance measurement of the microPET Focus 120 scanner. J Nucl Med. 2007;48:1527-1535.

24. Holland JP, Caldas-Lopes E, Divilov V, et al. Measuring the pharmacokinetic effects of a novel Hsp90 inhibitor on HER2/neu expression in mice using ${ }^{89} \mathrm{Zr}$ DFO-trastuzumab. PLoS One. 2010;5:e8859.
25. Guide for the Care and Use of Laboratory Animals. Washington, DC: National Academy Press; 1996.

26. Levin CS, Hoffman EJ. Calculation of positron range and its effects on the fundamental limit of positron emission tomography system spatial resolution. Phys Med Biol. 1999;44:781-799.

27. Cherry SR, Sorenson JA, Phelps ME. Physics in Nuclear Medicine. 3rd ed. Philadelphia, PA: Saunders; 2003.

28. McDevitt MR, Scheinberg DA. Ac-225 and her daughters: the many faces of Shiva. Cell Death Differ. 2002;9:593-594.

29. McDevitt MR, Ma D, Lai LT, et al. Tumor therapy with targeted atomic nanogenerators. Science. 2001;294:1537-1540.

30. Singh Jaggi J, Henke E, Seshan SV, et al. Selective alpha-particle mediated depletion of tumor vasculature with vascular normalization. PLoS One. 2007;2: e267.

31. Ballangrud ÅM, Yang W-H, Palm S, et al. Alpha-particle emitting atomic generator (actinium-225)-labeled trastuzumab (Herceptin) targeting of breast cancer spheroids: efficacy versus HER2/neu expression. Clin Cancer Res. 2004; 10:4489-4497.

32. Liu H, Moy P, Kim S, et al. Monoclonal antibodies to the extracellular domain of prostate-specific membrane antigen also react with tumor endothelium. Cancer Res. 1997;57:3629-3634.

33. Liu H, Rajasekaran AK, Moy P, et al. Constitutive and antibody-induced internalization of prostate-specific membrane antigen. Cancer Res. 1998;58: 4055-4060.

34. Smith-Jones PM, Vallabahajosula S, Goldsmith SJ, et al. In vitro characterization of radiolabeled monoclonal antibodies specific for the extracellular domain of prostate-specific membrane antigen. Cancer Res. 2000;60:5237-5243.

35. Smith-Jones PM, Vallabhajosula S, Navarro V, Bastidas D, Goldsmith SJ, Bander NH. Radiolabeled monoclonal antibodies specific to the extracellular domain of prostate-specific membrane antigen: preclinical studies in nude mice bearing LNCaP human prostate tumor. J Nucl Med. 2003;44:610-617.

36. McDevitt MR, Barendswaard E, Ma D, et al. An $\alpha$-particle emitting antibody $\left(\left[{ }^{213} \mathrm{Bi}\right] \mathrm{J} 591\right)$ for radioimmunotherapy of prostate cancer. Cancer Res. 2000;60: 6095-6100.

37. Verel I, Visser GWM, Boellaard R, et al. Quantitative ${ }^{89} \mathrm{Zr}$ immuno-PET for in vivo scouting of ${ }^{90} \mathrm{Y}$-labeled monoclonal antibodies in xenograft-bearing nude mice. J Nucl Med. 2003;44:1663-1670.

38. Perk LR, Visser OJ, Stigter-van Walsum M, et al. Preparation and evaluation of ${ }^{89} \mathrm{Zr}$-Zevalin for monitoring of ${ }^{90} \mathrm{Y}$-Zevalin biodistribution with positron emission tomography. Eur J Nucl Med Mol Imaging. 2006;33:1337-1345.

39. Borjesson PKE, Jauw YWS, de Bree R, et al. Radiation dosimetry of ${ }^{89} \mathrm{Zr}$ labeled chimeric monoclonal antibody U36 as used for immuno-PET in head and neck cancer patients. J Nucl Med. 2009;50:1828-1836.

40. Aerts HJWL, Dubois L, Perk L, et al. Disparity between in vivo EGFR expression and ${ }^{89} \mathrm{Zr}$-labeled cetuximab uptake assessed with PET. J Nucl Med. 2009;50:123-131.

41. Dijkers ECF, Kosterink JGW, Rademaker AP, et al. Development and characterization of clinical-grade ${ }^{89} \mathrm{Zr}$-trastuzumab for HER2/neu immunoPET imaging. J Nucl Med. 2009;50:974-981. 